\title{
Application of Bayesian Multilevel Modeling in Quantitative Structure-Retention Relationship Studies of Heterogeneous Compounds
}

\author{
Paweł Wiczling*, Agnieszka Kamedulska, Łukasz Kubik \\ Department of Biopharmaceutics and Pharmacodynamics, Medical University of Gdańsk, Gen. J. Hallera 107, 80-416 \\ Gdańsk, Poland
}

\section{Table of content:}

Table S1. Summary of the MCMC Simulations of the Marginal Posterior Distributions of Population-Level Model Parameters Figure S1. Relationship between the logarithm of retention factor $(\log \mathrm{k})$ and acetonitrile content in the mobile phase

Figure S2. Graphical display of the marginal posterior distributions for the effects of each functional group on $\pi_{\text {logkw, }} \pi_{\text {logka }}, \pi_{\text {log } S 2}, \pi_{\text {logkw }}-\pi_{\text {logka }}$ Figure S3. Scatter plots between individual chromatographic parameters, eta values (difference between the analyte-specific chromatographic parameter and expected value) and molecular mass

Figure S4. Effect of molecular mass on retention of compounds without functional groups

Figure S5. Comparison of model parameters obtained using the two-stage approach and multilevel model

Figure S6. Histogram of mean posterior values of the effects of each functional group on chromatographic parameters

Figure S7. Goodness-of-fit plots

Figure S8. Predictions for a random set of 10 analytes. 
Table S1. Summary of the MCMC Simulations of the Marginal Posterior Distributions of Population-Level Model Parameters.

\begin{tabular}{|c|c|c|c|c|c|c|c|c|}
\hline Parameter & Mean & MCSE & StdDev & $5 \%$ & $50 \%$ & $95 \%$ & N_Eff & R_hat \\
\hline$\theta_{\log k w}$ & 7.10 & 0.02 & 0.17 & 6.80 & 7.10 & 7.40 & 120 & 1 \\
\hline$\theta_{\log k a}$ & 0.05 & 0.00 & 0.07 & -0.07 & 0.05 & 0.17 & 350 & 1 \\
\hline$\theta_{\log S 2}$ & 0.38 & 0.00 & 0.02 & 0.34 & 0.38 & 0.42 & 270 & 1 \\
\hline$\beta_{\log k w}$ & 2.60 & 0.01 & 0.08 & 2.50 & 2.60 & 2.80 & 150 & 1 \\
\hline$\beta_{\log k a}$ & 0.40 & 0.00 & 0.03 & 0.35 & 0.40 & 0.45 & 350 & 1 \\
\hline$\beta_{\log S 2}$ & -0.02 & 0.00 & 0.01 & -0.04 & -0.02 & 0.00 & 370 & 1 \\
\hline$\omega_{\log k w}$ & 0.73 & 0.00 & 0.04 & 0.67 & 0.73 & 0.78 & 170 & 1 \\
\hline$\omega_{\log k a}$ & 0.33 & 0.00 & 0.02 & 0.30 & 0.32 & 0.35 & 270 & 1 \\
\hline$\omega_{\log S 2}$ & 0.12 & 0.00 & 0.01 & 0.11 & 0.12 & 0.13 & 260 & 1 \\
\hline$\rho_{[1,2]}$ & 0.62 & 0.00 & 0.03 & 0.57 & 0.62 & 0.67 & 520 & 1 \\
\hline$\rho_{[1,3]}$ & -0.14 & 0.00 & 0.05 & -0.23 & -0.14 & -0.06 & 150 & 1 \\
\hline$\rho_{[2,3]}$ & -0.28 & 0.00 & 0.05 & -0.35 & -0.28 & -0.20 & 400 & 1 \\
\hline$v$ & 2.30 & 0.01 & 0.20 & 2.00 & 2.30 & 2.60 & 290 & 1 \\
\hline$\sigma_{\pi-\log k w}$ & 0.83 & 0.01 & 0.10 & 0.68 & 0.82 & 0.99 & 270 & 1 \\
\hline$\sigma_{\pi-d l o g k}$ & 0.25 & 0.00 & 0.05 & 0.17 & 0.25 & 0.34 & 200 & 1 \\
\hline$\sigma_{\pi-\log S 2}$ & 0.07 & 0.00 & 0.01 & 0.06 & 0.07 & 0.09 & 340 & 1 \\
\hline$\theta_{\pi-\log k w}$ & 0.50 & 0.01 & 0.06 & 0.40 & 0.50 & 0.61 & 160 & 1 \\
\hline$\theta_{\pi-d l o g k}$ & 0.38 & 0.00 & 0.05 & 0.30 & 0.38 & 0.47 & 140 & 1 \\
\hline$v_{\pi}$ & 10.00 & 0.49 & 9.80 & 2.30 & 7.00 & 30.00 & 400 & 1 \\
\hline$\sigma$ & 0.01 & 0.00 & 0.00 & 0.01 & 0.01 & 0.01 & 400 & 1 \\
\hline Vobs & 1.30 & 0.00 & 0.05 & 1.20 & 1.30 & 1.40 & 500 & 1 \\
\hline
\end{tabular}

Mean denotes sample mean, MCSE denotes Monte Carlo Standard Error, StdDev denotes sample standard deviation, $5 \%$, $\mathbf{5 0} \%, \mathbf{9 5} \%$ denote corresponding quantiles, N_Eff denotes effective sample size, R_Hat denotes a measure of chain equilibrium, must be within 0.05 of 1.0 . 




Figure S1. Relationship between the logarithm of retention factor (log k) and acetonitrile content in the mobile phase. Lines connect measurements obtained for a particular analyte. 


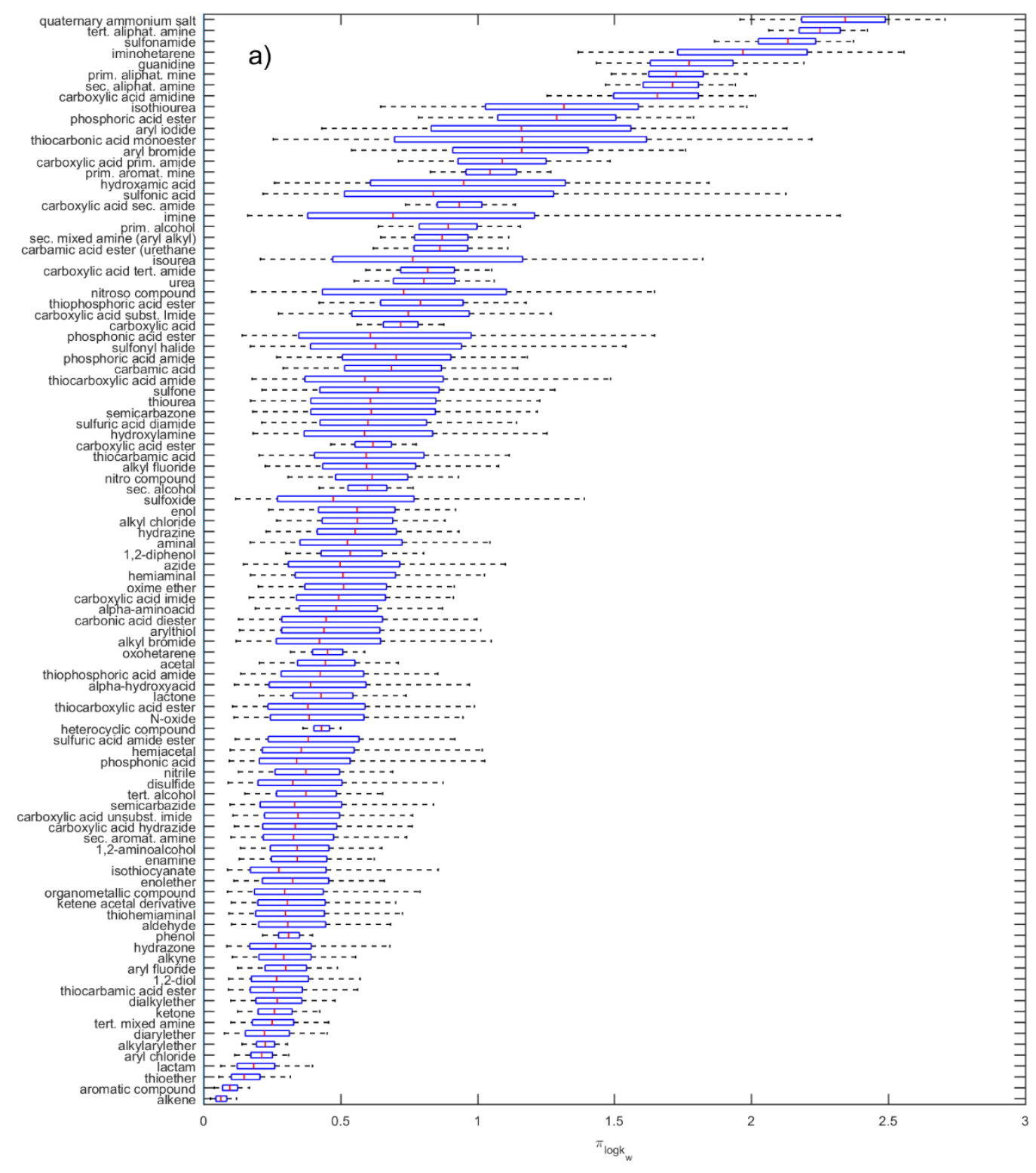




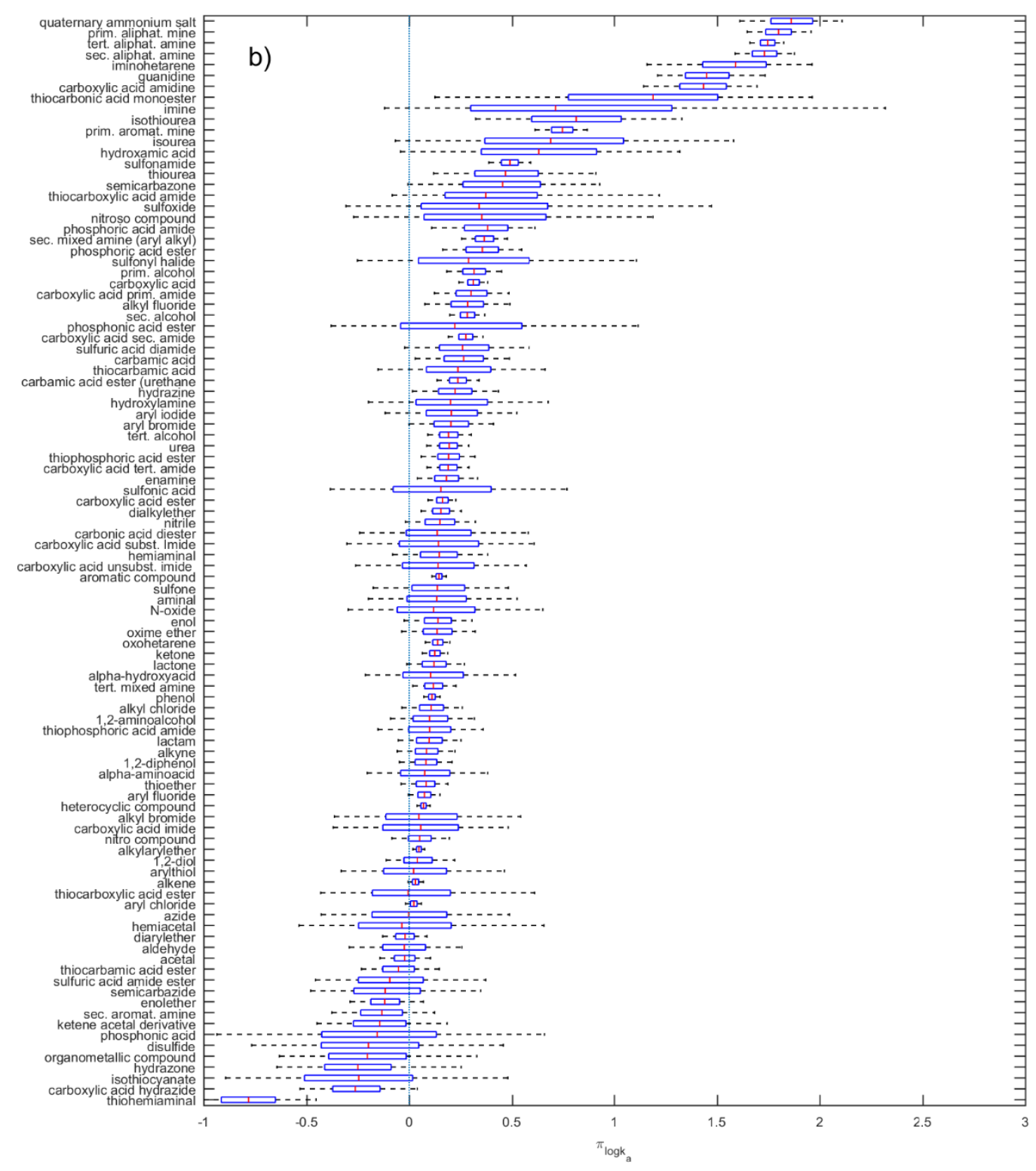




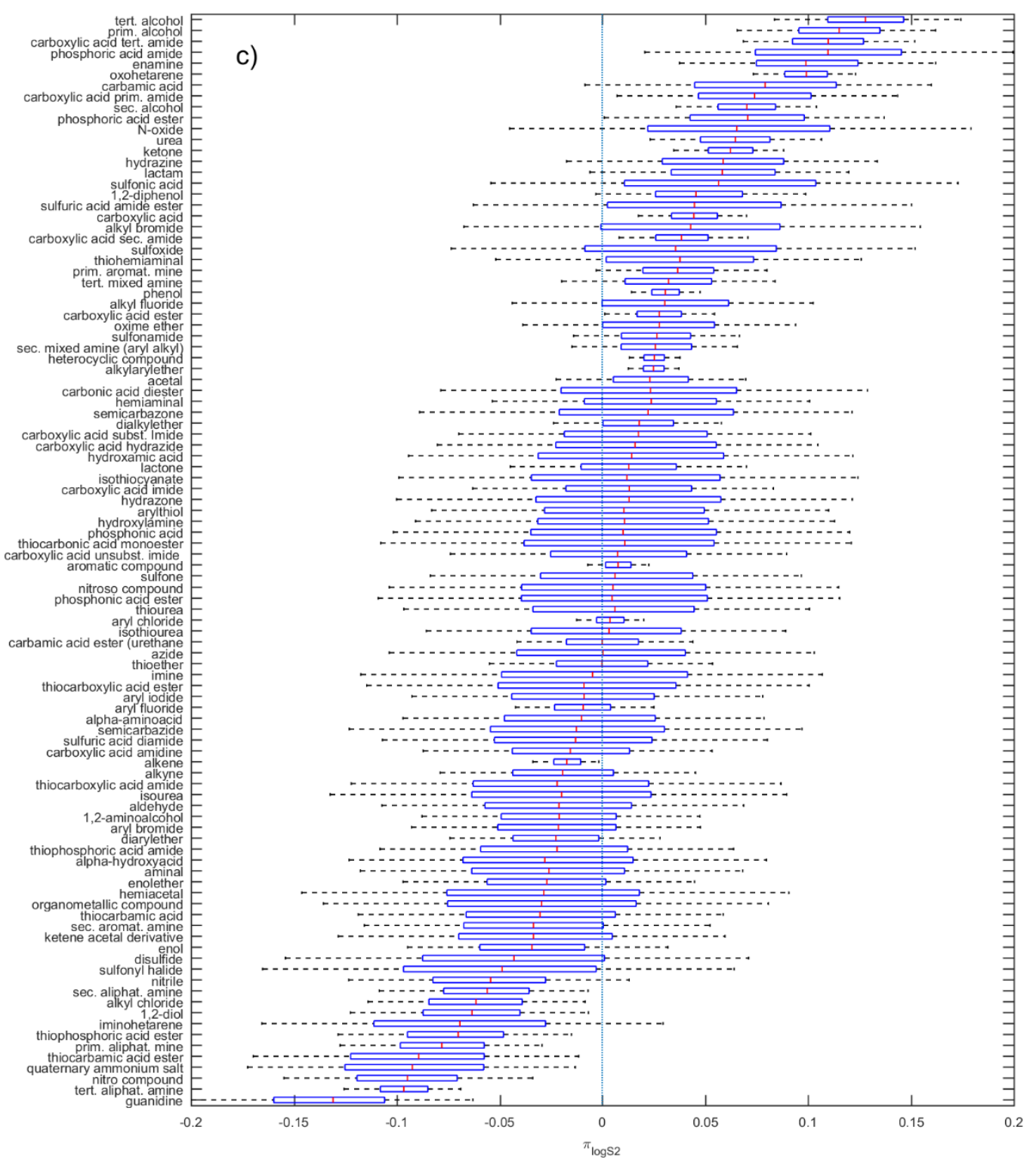




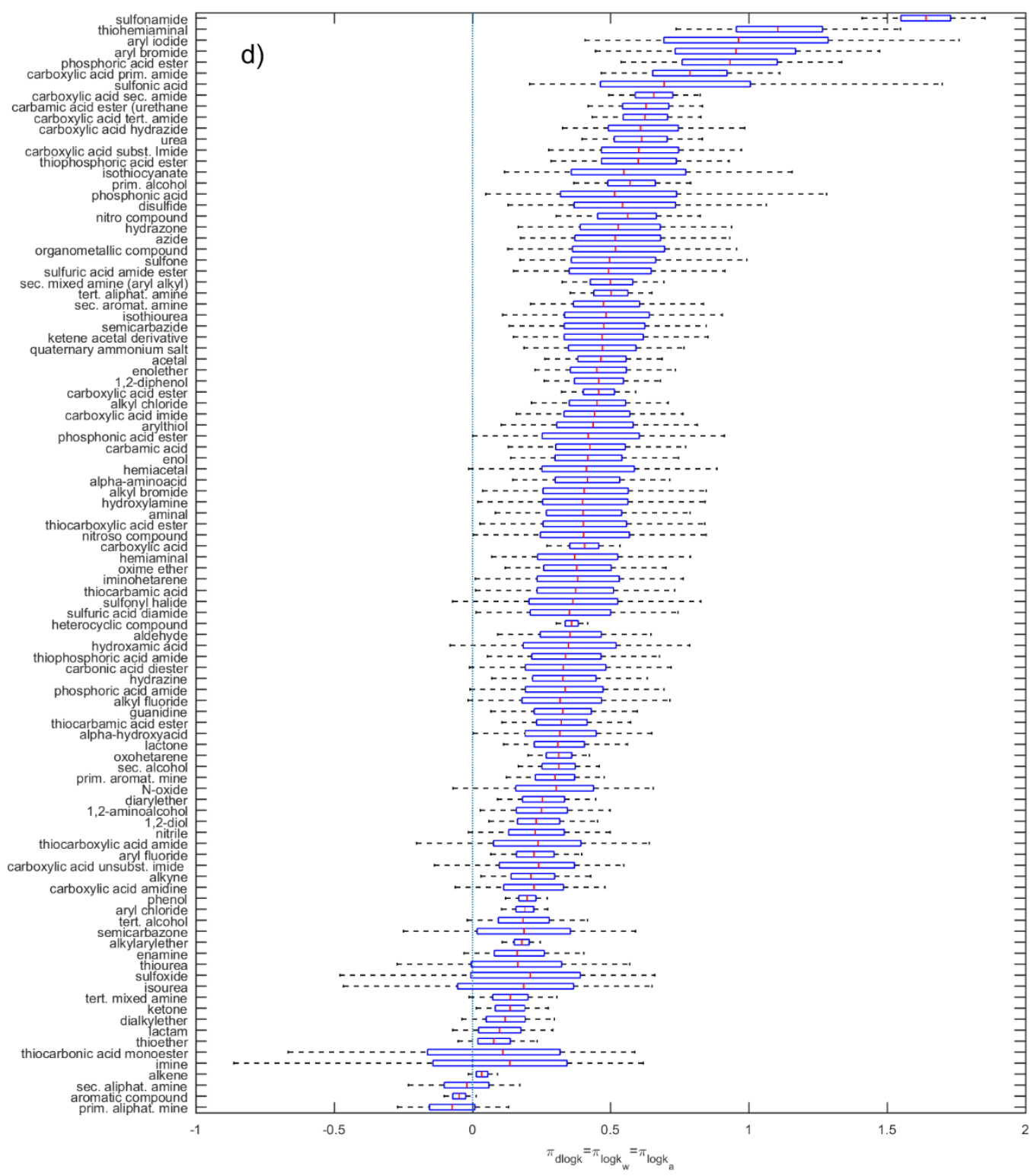

Figure S2. Graphical display of the marginal posterior distributions for the effects of each functional group on a) $\pi_{\log k w}$, b) $\pi_{\log k a}$, c) $\pi_{\log S 2}$, and d) the difference between $\pi_{\text {logkw }}$ and $\pi_{\text {logka }}$. 
a)

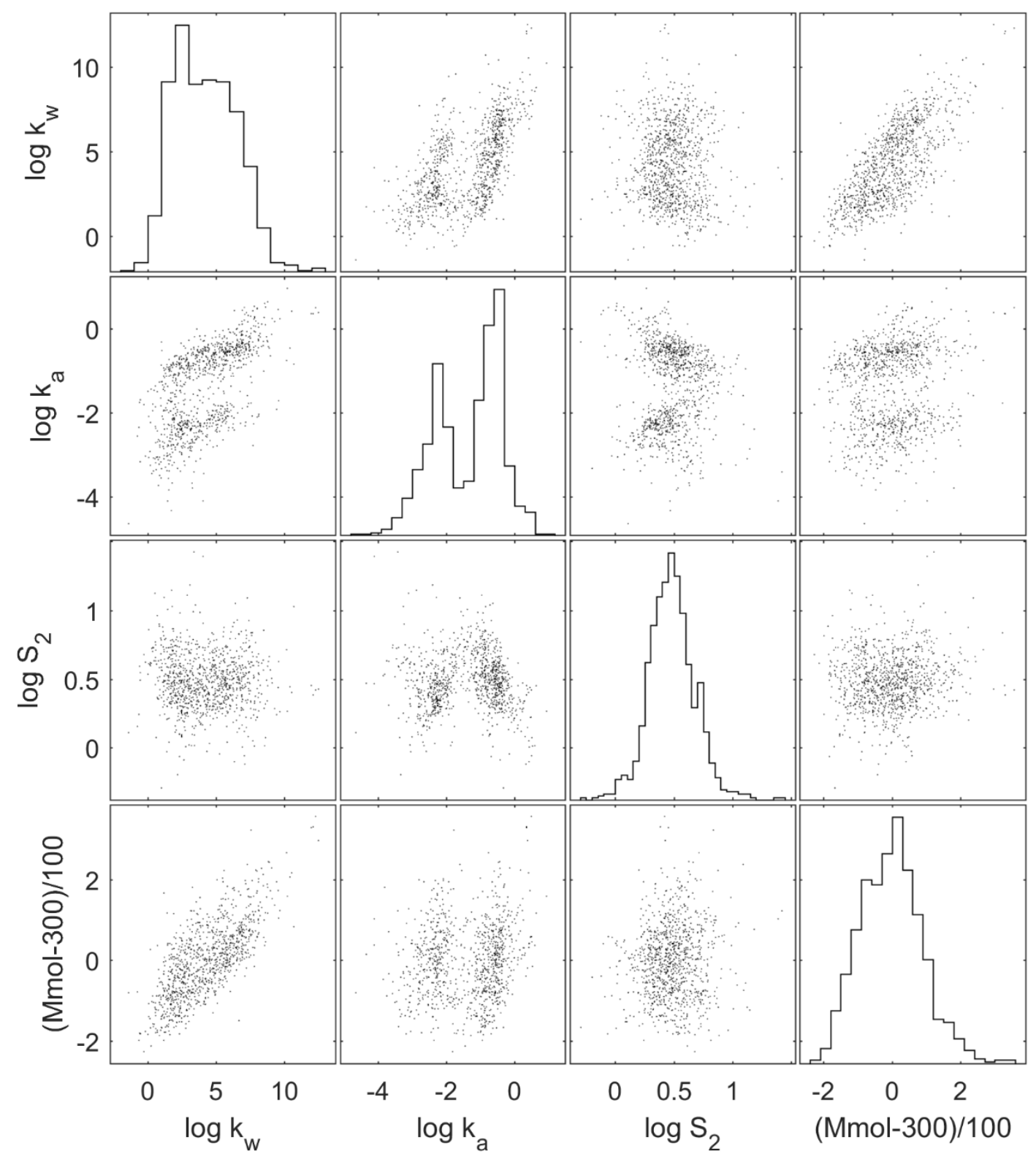


b)

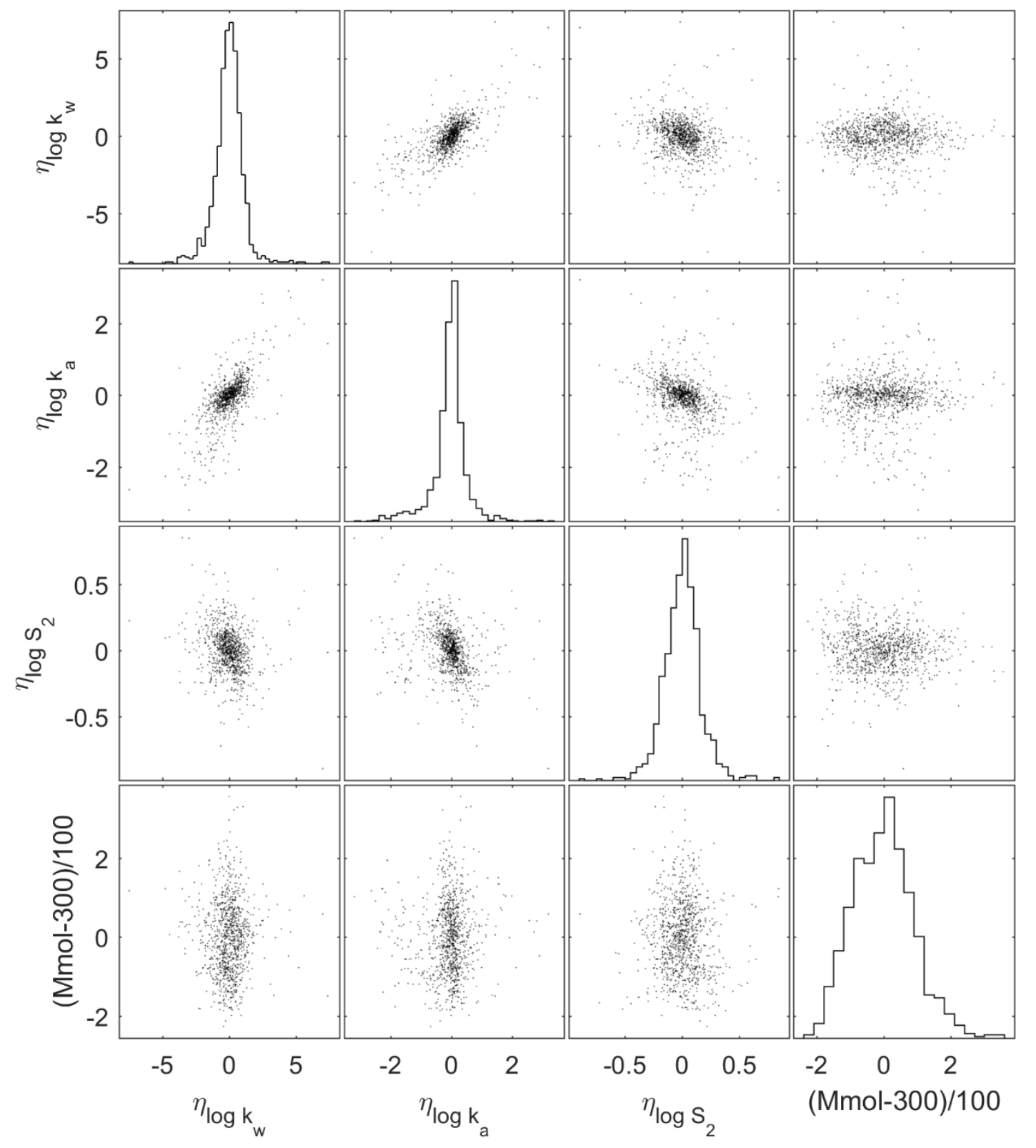

Figure S3. Scatter plots between a) individual chromatographic parameters and molecular mass, and b) eta values (difference between the analyte-specific chromatographic parameter and expected value) and molecular mass. Diagonal subplots present histograms. 

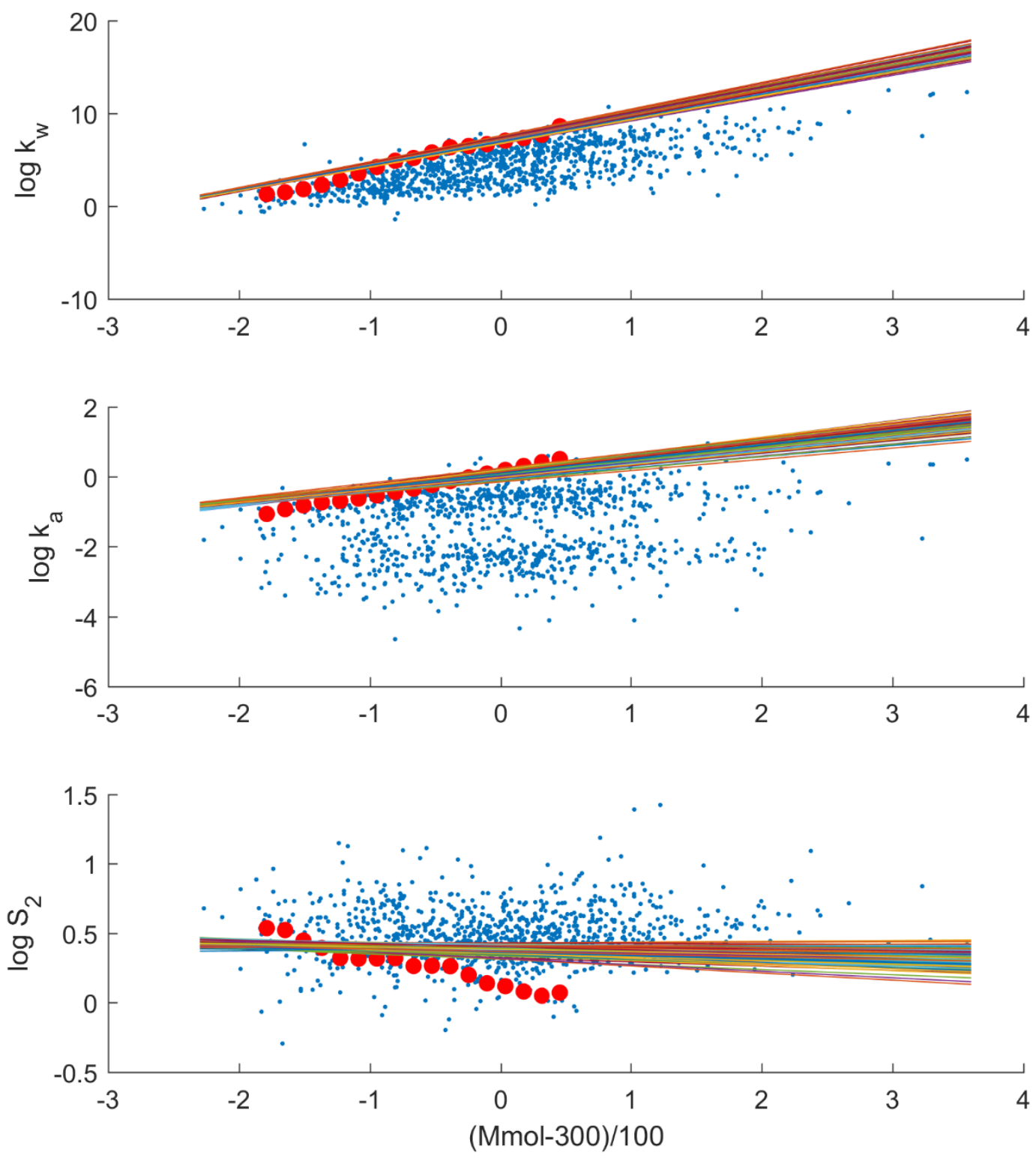

Figure S4. Effect of molecular mass on retention of compounds without functional groups (10 draws of the expected line). The analytespecific chromatographic parameters are lower due to the presence of functional groups. For $\log S_{2}$, the effects are in both directions. A congeneric series of alkyl-substituted benzamides (benzamide and N-methyl-, N-ethyl, ..., N-pentadecyl-, N-hexadecyl benzamides) is shown to highlight the effect of molecular mass. 

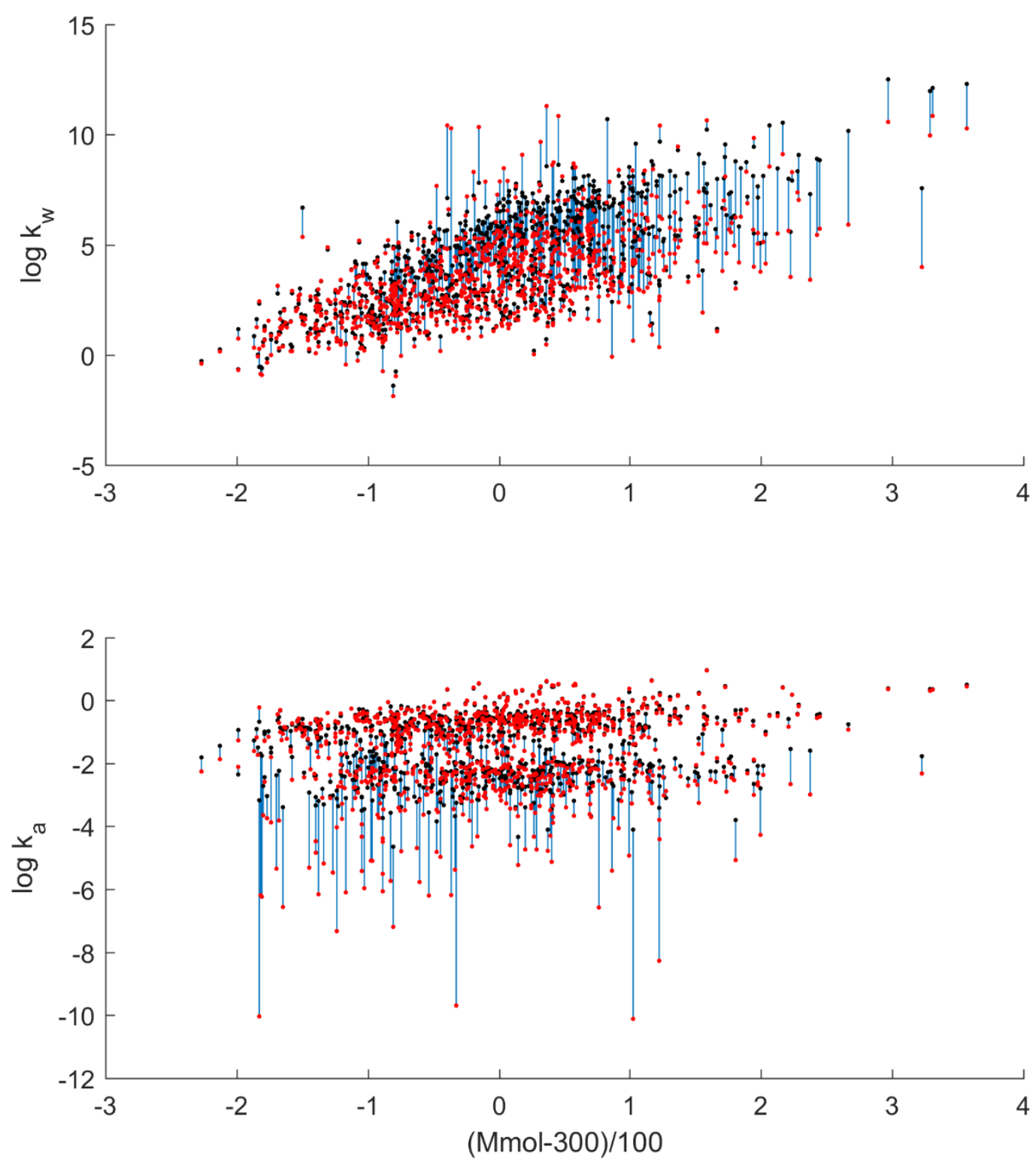

Figure S5. Comparison of model parameters obtained using the two-stage approach and multilevel model. Shrinkage is shown as lines connecting the parameters estimated by both methods. The multilevel model shows more stable estimates, particularly for parameters that are difficult to estimate precisely due to lack of information. 

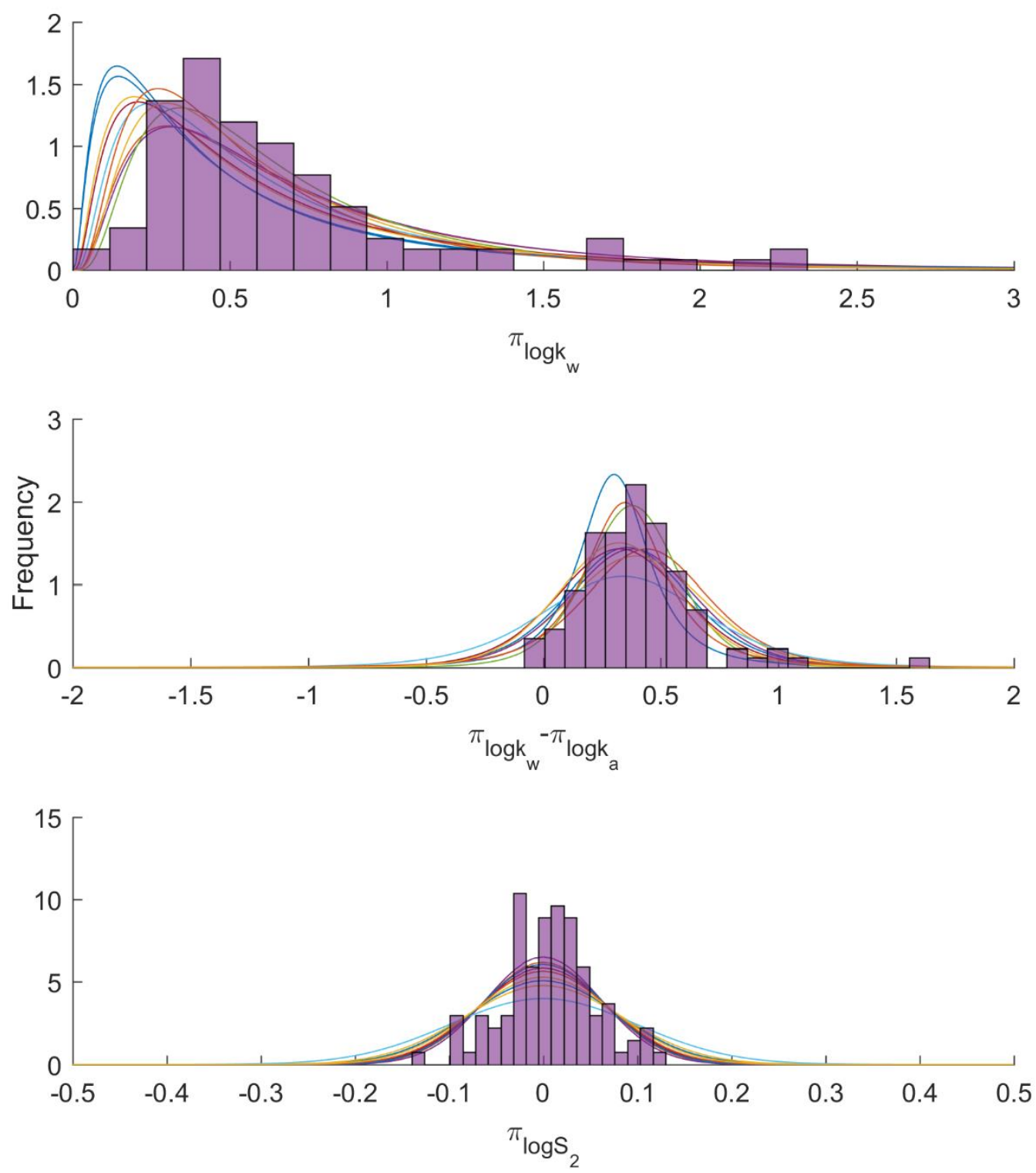

Figure S6. Histogram of mean posterior values of the effects of each functional group on chromatographic parameters. 

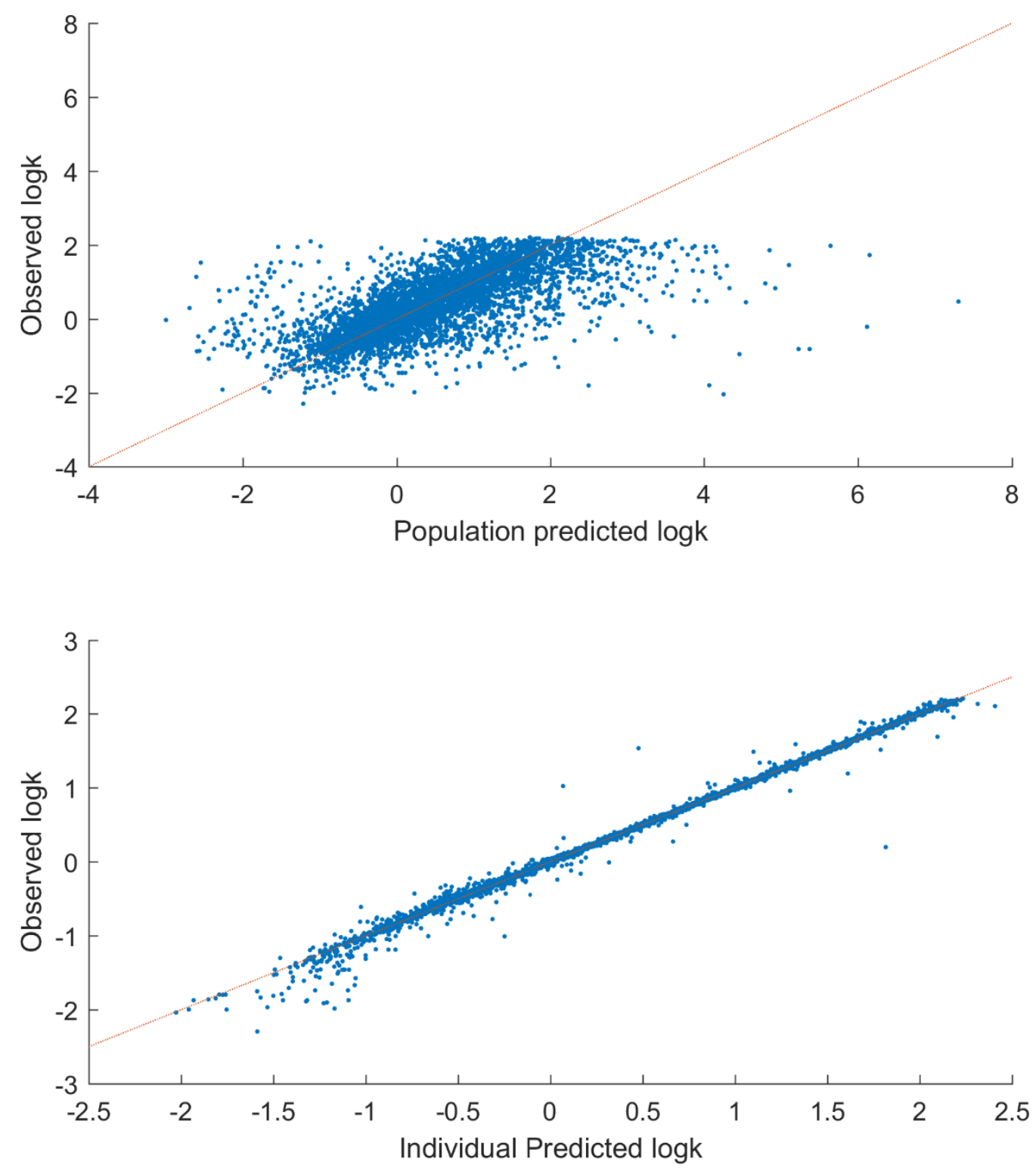

Figure S7. Goodness-of-fit plots. The observed vs. the mean population-predicted retention factors (i.e., the a posteriori means of predictive distributions corresponding to the future observations of a new analyte) and the observed vs the mean individual-predicted retention times (i.e., the a posteriori mean of a predictive distribution conditioned on the observed data from the same analyte). 

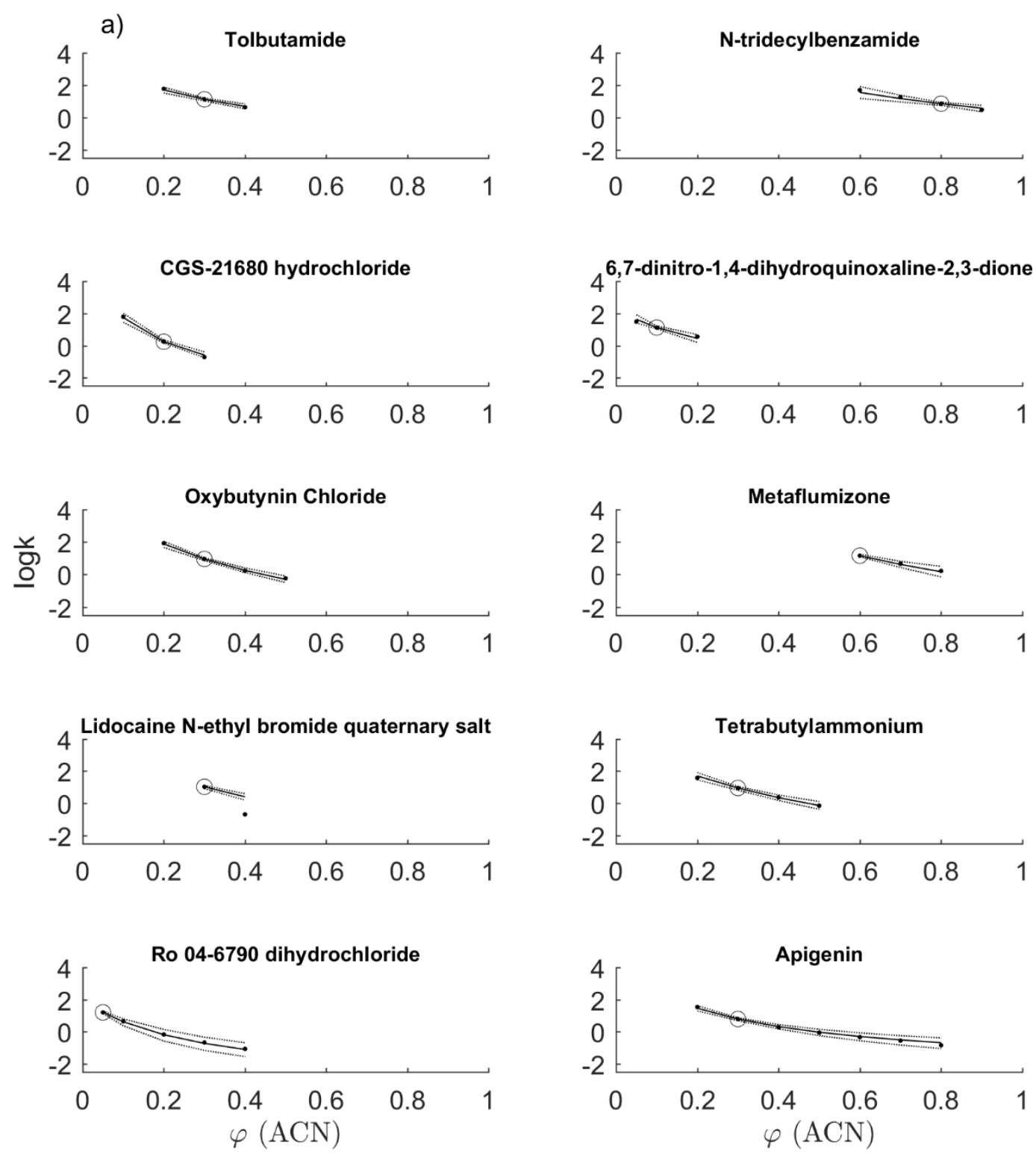

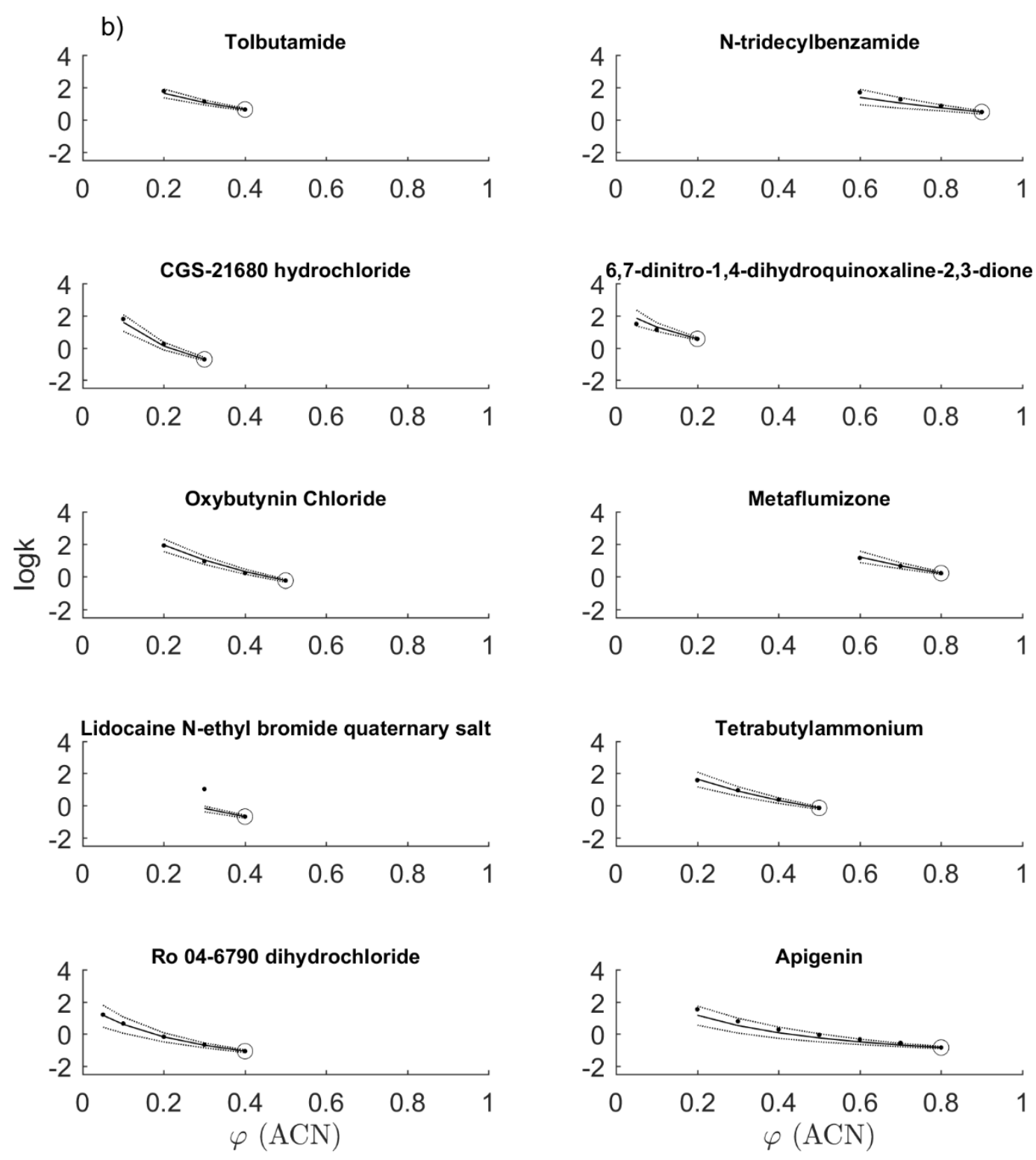

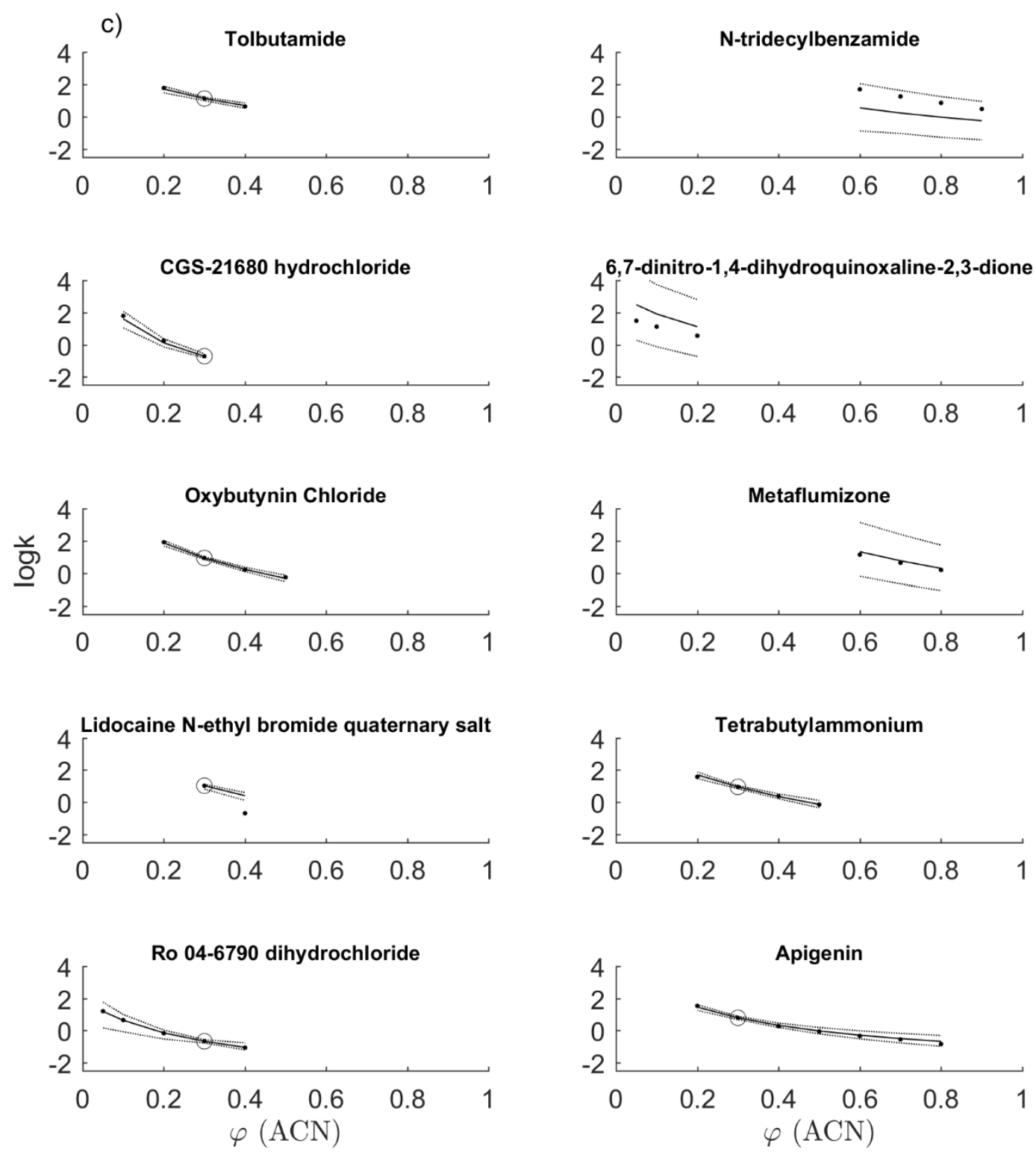

Figure S8. Predictions represented as posterior median (line) and $5^{\text {th }}-95^{\text {th }}$ percentiles (dotted lines) for a random set of 10 analytes. Observed retention factors are shown as dots. Predictions corresponding to future isocratic observations given single retention time measurements are shown as an open symbol. The preliminary data correspond to a) isocratic measurements with $\log k$ equal approximately 1 , b) isocratic measurement with minimum observed value of $\log k$ for a particular analyte, c) one isocratic measurement conducted at $\varphi=0.3$. 